\title{
A investigação sobre o ensino dos jogos desportivos: ensinar e aprender as habilidades básicas do jogo
}

\author{
A.S. Graça \\ I.R. Mesquita
}

\author{
Faculdade de Ciências do Desporto e de Educação Física \\ Universidade do Porto
}

\section{RESUMO}

O presente estudo faz uma revisão da evolução da investigação sobre o ensino dos jogos desportivos, delimitando a sua abrangência à investigação relativa ao ensino e aprendizagem das habilidades básicas, constituindo esta tarefa apenas uma parte, mas a mais preponderante, do esforço de investigação nesta temática. A uma fase inicial, marcada pelas questões oriundas da aprendizagem motora, muito dependente dos estudos ligados à realização de provas académicas, caricaturada na infindável guerra dos métodos, sucedeu uma fase de observação sistemática do processo de ensino e aprendizagem, com relevo especial para os estudos focados sobre o empenhamento dos alunos na aula e a sua relação com o sucesso na aquisição das habilidades. Os sucessivos esforços de qualificação e refinamento das variáveis de empenhamento do aluno, a par de um crescente deslocamento das bases conceptuais da esfera de influência das perspectivas behavioristas para as cognitivistas aproximaram as questões do estudo da busca de um entendimento mais íntimo da natureza do trabalho do aluno, dos objectivos, dos conteúdos e dos contextos das tarefas e do nível de processamento dos alunos no confronto com essa tarefas. A organização dos contextos de prática tem merecido nas últimas décadas uma atenção especial, procurando-se novamente uma ligação às ideias da aprendizagem motora, como é o caso da teoria da interferência contextual, ainda que os resultados não se apresentem tão inequívocos. A atenção dada à realização das habilidades por diversos programas parece, por vezes, resultar e também, porventura, induzir uma visão fragmentada e descontextualizada das habilidades dos jogos.

Palavras-chave: jogos desportivos, habilidades, ensino, revisão da investigação

\author{
ABSTRACT \\ Research on teaching sport games: \\ Teaching and learning games skills
}

This study reviews the evolution of the research on the sport games related to the issues of teaching and learning the sport games skills, which is no more than a part of the research effort in the domain of sport games, yet the prominent one. In the first phase the research question was rooted upon motor learning topics, and the studies confined to academic graduation requirements. It was the time of the "methods war". A new phase began with the systematic observation of the teaching and learning process, with a particular interest on learner engagement and the correlated effect on skill achievement. The continued efforts to qualify and refine learner engagement variables, along with a growing move from a behaviourist to a cognitivist perspective has put the research questions more and more closed to the intimacies of the student work, to the tasks goals, content and contexts, and to the level of the learner processing on those tasks. In the last decades, the arrangement of the contexts for practice has deserved special attention by renewing the connection with the motor learning topics, such as the contextual interference, although results were not so conclusive. The attention the programs usually assigned to skill execution may be both a result of and a cause to a fragmented and non-contextualized view of the sport games' skills.

Keywords: Sport games, games' skills, teaching, research review 


\section{INTRODUÇÃO}

Os jogos desportivos ocupam um lugar dominante nos programas de Educação Física das mais diversas latitudes, na Austrália (7), no Canadá (26), nos Estados Unidos (43), na Inglaterra (11). Os jogos desportivos ocupam igualmente um lugar cimeiro no conjunto das actividades organizadas em que participam crianças e jovens. Porém, se tomarmos a revista JTPE como uma boa janela para apreciar o panorama da investigação empírica na área da pedagogia do desporto, podemos verificar que em 20 anos de publicação apenas 54 em 613 títulos de artigos remetem explicitamente para os jogos desportivos, o que nos pode dar a ideia de que o ensino dos jogos tem sido uma realidade pouco estudada.

Tradicionalmente, as práticas de ensino dos jogos colocam a aprendizagem das habilidades como um pré-requisito para o sucesso da participação no jogo. As progressões didácticas concentram-se básica ou exclusivamente nos aspectos da execução motora das habilidades básicas em situações descontextualizadas. A investigação sobre o ensino do jogo, de uma forma clara, ou então por omissão ou conveniência, tem predominantemente assumido o primado do domínio das habilidades.

Nos anos mais recentes, as perspectivas cognitivistas e construtivistas recentraram ou alargaram as abordagens e a investigação sobre os jogos a domínios tradicionalmente negligenciados, como sejam o domínio da táctica e da sua interacção complexa com a técnica, envolvendo o conhecimento, a compreensão, a tomada de decisão e a capacidade de acção em situação de jogo.

Uma revisão sobre o ensino do jogo deve procurar, ainda que aqui se faça de forma compreensiva, traçar o panorama da evolução da investigação sobre o tema, apontando as questões fundamentais, o modo como foram operacionalizadas, os resultados mais relevantes e as principais críticas, debilidades e impasses.

Por razões de espaço limitaremos a revisão à investigação que tematiza ou se desenvolve no pressuposto do primado das habilidades básicas do jogo, deixando para futura publicação a revisão da investigação que contempla outros domínios relacionados com o ensino e aprendizagem dos jogos desportivos.

A estrutura do artigo desenvolve-se em 5 pontos. Ela deixa perceber uma lógica de sucessão de fases de investigação entre os diversos pontos, mas também em certo grau a coexistência de ramos de investigação consideravelmente distintos. O primeiro ponto, que designamos de primeiros passos ou falsas parti- das, remete para um tempo de investigação marcado essencialmente pela comparação de métodos de instrução. O segundo ponto representa um corte com a investigação anterior e refere-se aos estudos de observação sistemática do comportamento de professores e alunos, incidindo particularmente nos estudos focados sobre o empenhamento motor dos alunos. O terceiro ponto lida com o esforço da investigação para ultrapassar o carácter genérico e algo grosseiro das variáveis de empenhamento do aluno e definir variáveis mais específicas e diferenciadas do desenvolvimento do conteúdo e delineamento das tarefas de aprendizagem. O quarto ponto contempla a investigação que procura testar os efeitos da interferência contextual na aprendizagem das habilidades dos jogos em condições de instrução não laboratoriais. Por último, o quinto apresenta um breve balanço da investigação revista e perspectiva a emergência de um questionamento para além do horizonte da execução das habilidades, entrando em linha de conta com outras dimensões fundamentais, em particular a dimensão cognitivo-táctica do jogo.

\section{PRIMEIROS PASSOS OU FALSAS PARTIDAS?}

No capítulo de Nixon e Locke (42) do $2^{\circ}$ Handbook of Research on Teaching, dedicado à revisão da investigação no ensino da Educação Física, pudemos enumerar 60 referências bibliográficas de estudos empíricos cujo título fazia menção explícita a diferentes tipos de jogos desportivos, com destaque para o ténis $(n=23)$, basquetebol $(n=18)$, badminton $(n=8)$ e voleibol $(n=6)$. A grande maioria destes estudos reporta-se à década de 60 e refere-se a provas de graduação académica, principalmente dissertações de mestrado $(n=33)$. A generalidade destes estudos enquadra-se numa fase da investigação do ensino que se centrou na descoberta do método ideal, através da comparação de resultados produzidos em uma ou mais habilidades pela aplicação experimental de diferentes métodos, procedimentos ou meios de ensino, ou formas de organização do currículo e dos alunos (por exemplo: método parcial vs. global; demonstração; treino mental; instrução directa vs. instrução programada; sequências de progressões; distribuições da prática; tamanhos da turma; agrupamento dos alunos por níveis de habilidade; coeducação vs. educação segregada; dimensões do objecto de jogo, dos engenhos ou equipamentos). Nesta fase, a investigação do ensino tinha alguma dificuldade em se diferenciar da investigação da área da aprendizagem motora, aparecendo como parente 
pobre duma área que crescia em especialização, mas que, para o fazer, se distanciava dos problemas práticos do ensino e da aprendizagem da aula de educação física ou do treino desportivo. Fica deste tempo uma intenção de testar e transpor para as condições do terreno da prática ilações e sugestões extraídas da investigação laboratorial da aprendizagem motora. Não teve porém grande sucesso e a generalidade das suas conclusões não teve qualquer repercussão ou impacto. A debilidade destes estudos e a crítica que sobre eles se abateu resulta sobretudo do carácter avulso das variáveis experimentais, dado que não apareciam escudadas nem em teorias de aprendizagem unificadoras, que as não havia, nem em teorias de ensino coerentes, isto para além das insuficiências metodológicas que hipotecavam a validade externa dos resultados. Como refere Locke (35), o número reduzido de turmas incluídas nos estudos e a adopção de unidades de análise inadequadas confundiam os efeitos eventualmente produzidos pelos métodos com efeitos atribuíveis a diferenças idiossincráticas dos professores. Talvez por tudo isto Siedentop e Tannheill (61) passem por cima de todos estes estudos e sinalizem o início da investigação em ensino com a publicação de "What's going on in gym", editado por Anderson e Barrette (3), que compila um conjunto de estudos de observação sistemática do comportamento de professores e alunos na aula de educação física.

\section{OBSERVAÇÃO SISTEMÁTICA DO ENSINO DO JOGO E DAS HABILIDADES}

Os estudos centrados na observação sistemática do ensino do jogo e das respectivas habilidades foram, numa primeira fase, de natureza descritiva.

Pretenderam fazer o retrato da forma como os professores e treinadores desempenhavam as diferentes funções de ensino, com especial relevo para o feedback pedagógico, e estruturavam e geriam as sessões de ensino do jogo e das habilidades, nomeadamente no respeitante ao tempo destinado à exercitação dos conteúdos e à participação dos alunos ou atletas nas actividades.

Merece ser assinalado o conjunto de trabalhos de uma equipa de investigadores finlandeses apresentado ao International Congress on Teaching Team Sports (1983, Roma), iniciativa que infelizmente não teve continuidade e que colocou lado a lado representantes das diversas comunidades interessadas no estudo dos jogos desportivos e no seu ensino. Varstala et al. (69) compararam o comportamento de professores e professoras e verificaram que os professores ensinavam os jogos mais frequentemente e dedicavam mais tempo a jogar e menos tempo a exercitar as habilidades do jogo que as professoras. Laakso et al. (29) reportam que os alunos do ensino secundário, de ambos os sexos, são capazes de aplicar habilidades básicas de voleibol em situações de jogo, mas com grandes diferenças entre eles. Telama et al. (65) compararam a participação de rapazes e raparigas do ensino secundário nas aulas de diferentes jogos desportivos. Rapazes e raparigas diferenciam-se nos comportamentos e padrões de actividade, havendo também diferenças de modalidade para modalidade, principalmente entre as raparigas.

O constructo Academic Learning Time in Physical Education (ALT-PE) assumiu particular relevância na investigação no ensino da Educação Física, dos finais dos anos 1970 aos primeiros anos de 1990. O ALTPE contabiliza o tempo individual do aluno passado em conteúdo específico de Educação Física com nível apropriado de dificuldade obtendo elevado grau de sucesso no desempenho das tarefas $(40,60)$. A associação, empiricamente verificada no ensino geral, entre o ALT e os ganhos de aprendizagem sugeriu que este constructo poderia constituir uma forma válida não só de ajuizar acerca da qualidade da aula ou da sessão de treino, como até de medir indirectamente a aprendizagem, o que no caso dos jogos desportivos, dada ausência de produtos permanentes, se tornava num substituto muito aliciante (45). A sustentabilidade desta hipótese viria a ser parcialmente confirmada num estudo de Silverman Devillier e Ramirez (62).

A maior parte dos estudos que analisaram as variáveis relacionadas com a oportunidade de resposta, no âmbito do ensino e treino dos jogos desportivos, nomeadamente o ALT-PE, ou mais genericamente o tempo de empenhamento motor, foram realizados no contexto escolar, sendo em menor escala os estudos realizados no âmbito do clube.

$\mathrm{O}$ tipo de actividade aparece como um factor marcante nas diferenças de tempo de empenhamento motor dos alunos. As aulas de jogos desportivos proporcionam substancialmente mais tempo de empenhamento motor que as de ginástica ou atletismo $(48,51)$, mas menos que as de dança (44). A comparação dos tempos de empenhamento motor dos alunos por sexo ou nível de habilidade não produziu evidência inequívoca. Os estudos de Shute et al. (59), Silverman et al. (1984), Pimentel (52) e Graça (18) não reportam diferenças significativas entre 
sexos ou entre níveis de habilidade. Mesquita (38) também não encontra diferenças significativas de tempo de empenhamento motor global entre jovens voleibolistas do sexo feminino, de diferentes níveis de habilidade, o que já não acontece no caso dos exercícios específicos quando analisados de forma agregada. Outros estudos, porém, referem vantagens dos rapazes $(63,64)$, ou dos alunos de nível elevado $(47,49)$, ou de atletas de nível mais elevado (36). Na comparação do empenhamento na aula de educação física e na sessão de treino desportivo, dedicadas ao ensino e treino de basquetebol, Piéron e Gonçalves (50) verificaram que o mesmo professor proporcionava o mesmo tempo de exercitação das habilidades do jogo, que era em ambos os casos o maior segmento da aula ou sessão de treino (cerca de $40 \%$ do tempo observado). No entanto, nas situações de jogo, o professor tinha uma actuação bastante diferente nos dois contextos. Na aula de educação física, a forma de jogo dominante era o $5 \times 5$, com as situações de jogo reduzido a ocuparem uma percentagem de tempo diminuta ( 21.1 vs. 4.6$)$, enquanto que no treino, o jogo $5 \times 5$ é suplantado pelas formas de jogo reduzido (14.9 vs. 19.2). Privilegiar formas de jogo bem mais complexas no contexto da aula de educação física do que no treino desportivo dificilmente se poderá encaixar numa lógica de progressão didáctica do ensino do jogo, embora pareça ser essa a forma tradicional de ensinar o jogo na escola (19). Aos estudos descritivos sucedem-se os correlacionais entre variáveis do processo de ensino e aprendizagem e uma medida válida de produto da aprendizagem, visando o entendimento dos factores que influenciam os ganhos da aprendizagem no ensino das actividades desportivas (41).

Dos estudos correlacionais ou comparativo-causais realizados no âmbito dos jogos desportivos, é de destacar o estudo de Phillips e Carlisle (46), aplicado no Voleibol, um dos estudos de maior envergadura na linha de investigação processo-produto e que pôs em evidência o efeito do tempo de empenhamento motor; em claro contraste situam-se os estudos de Brunelle et al. (8) e de Godbout, Brunelle \& Tousignant (16), aplicados a jovens atletas e realizados em diferentes modalidades colectivas (Hóquei no gelo, Voleibol e Andebol), onde a relação entre a oportunidade de resposta e os ganhos de aprendizagem não se evidenciou.

Os esforços para credibilizar o ALT-PE como variável preditiva dos ganhos de aprendizagem dos alunos não foram concludentes (57). Podemos entender este constructo como uma variável necessária mas não suficiente para a realização das aprendizagens, na medida em que praticar as tarefas com sucesso num tempo de exercitação apropriado não significa que a qualidade da prática seja suficiente para que se verifique melhoria na performance, ou mesmo que a tarefa esteja de acordo com os objectivos da aprendizagem $(1,57)$.

Mesmo no ensino geral, onde a associação entre tempo de empenhamento do aluno e resultados de aprendizagem foi bem estabelecida, se criticou a ênfase neste tipo de medidas, nomeadamente pela não consideração da especificidade do conteúdo, da qualidade da aprendizagem, da qualidade do trabalho do aluno, e por espelhar uma visão fragmentária e descontextualizada das matérias, dos conhecimentos e das habilidades (53).

Com base nas características que definem os jogos desportivos colectivos, Parker e O'Sullivan (45) justificaram a pertinência de substituir ou complementar a observação de variáveis temporais por variáveis discretas (número de execuções), nos casos em que fosse manifesta a vantagem de utilizar a frequência de respostas correctas como variável critério, porquanto a análise de variáveis discretas no contexto dos jogos desportivos colectivos permitia ter em conta as condições e os critérios de realização das respostas. A intenção era aceder a um conhecimento mais pormenorizado do envolvimento e consequentemente à possibilidade de determinação dos tipos de envolvimento que proporcionam elevadas frequências de execução das habilidades dos jogos desportivos (38).

Buck e Harrison (9), Buck, Harrison e Bryce (10) estudaram o ensino do voleibol na escola, com o propósito de analisar a qualidade e quantidade de execuções das quatro habilidades básicas do jogo (passe, manchete, serviço e remate), ao longo de 22 aulas, e relacioná-las com os ganhos de aprendizagem. Nas duas turmas observadas, o jogo formal ocupou mais de metade do tempo dedicado à prática. De uma forma geral, todos os alunos realizaram poucos contactos com a bola, e os de nível baixo praticamente não contactaram com ela durante o jogo. A análise da relação entre o número de execuções das habilidades e os ganhos de aprendizagem põe em evidência a influência determinante do número de execuções correctas e sublinha também a influência do nível inicial. Com base nestas constatações, os autores salientam a necessidade de ser dada mais atenção ao ensino das habilidades complexas que requerem coordenação e ajustamento temporal; 
os alunos de nível de desempenho baixo necessitam de situações de aprendizagem mais estruturadas que possibilitem não só o aumento do número total de contactos, como também a ocorrência de maior qualidade de resposta. A quase total ausência de sucesso registada pelos alunos de baixo nível põe em causa a utilização do jogo formal como meio preferencial de aprendizagem do Voleibol e sugere uma articulação mais funcional da exercitação das habilidades específicas com formas de jogo reduzido.

Mesquita (38) realizou um estudo centrado na mesma problemática com aplicação no clube. Teve como propósito comparar a frequência de resposta e a taxa de sucesso entre jogadoras de nível superior e médio na exercitação das habilidades técnicas, estendendo a análise comparativa às situações de treino e de competição. Durante dois microciclos, que perfizeram 10 treinos, observou duas equipas do escalão de iniciados feminino; simultaneamente foram observados 4 jogos contra um adversário comum, mais 2 jogos entre as equipas em estudo. A autora constatou que nos exercícios com estrutura semelhante à do jogo as jogadoras de nível superior registaram frequências de resposta significativamente superiores, acentuando-se as diferenças entre os dois grupos na qualidade de resposta. A competição acentuou as diferenças registadas no treino entre os dois grupos, na quantidade e na qualidade de resposta ao nível das habilidades técnicas (passe, manchete, serviço e remate). Perante estes resultados a autora enfatiza a necessidade do ensino e treino das habilidades técnicas ser estruturado de uma forma progressiva e sequencial em referência às exigência do jogo, preconizando o refinamento das tarefas nas aprendizagens mais exigentes.

\section{DESENVOLVIMENTO DO CONTEÚDO \\ E DELINEAMENTO DAS TAREFAS DE APRENDIZAGEM}

Housner (27) analisou o modelo de ensino "direct instruction", sintetizado a partir das conclusões dos principais estudos de investigação processo-produto, as quais evidenciaram as variáveis relativas aos comportamentos de instrução dos professores e ao trabalho académico que aparecem associadas de forma consistente a efeitos importantes nos resultados de aprendizagem dos alunos. O autor critica, porém, as limitações do modelo, referindo que ele é eficaz no ensino de conteúdos bem estruturados, mas não tanto com conteúdos mais difíceis de decompor para um tipo de abordagem passo-a-passo, que exija resolução de problemas, interpretação de informação não familiar ou aplicação de regras e leis a situações novas. $\mathrm{O}$ autor destaca ainda a insuficiência explicativa das medidas de empenhamento do aluno, considerando a necessidade de incluir variáveis de mediação com destaque para o empenhamento cognitivo e as estratégias de processamento do aluno.

Os resultados do estudo de Carreiro da Costa (12) sublinham bem a necessidade de diferenciar e especificar o conteúdo da participação motora e cognitiva em função das necessidades dos alunos e dos objectivos visados. A relação entre a prática do estudante e os objectivos da aprendizagem devem ser considerados, com especial incidência nas habilidades motoras complexas $(9,57)$, como é o caso dos jogos desportivos colectivos, aonde coexistem múltiplas escolhas na resolução das tarefas.

Com base neste entendimento, a investigação procurou identificar os caminhos pelos quais o professor, ou treinador, consegue produzir qualidade nas respostas dos alunos em conformidade com os objectivos definidos. A selecção das tarefas por parte do professor ou do treinador constitui um dos elementos com especial interesse para os investigadores, uma vez que elas são intermediárias entre os objectivos da aprendizagem e as respostas dos alunos (57). Graham (20) realizou um estudo com particular interesse, ao analisar de forma sequencial as tarefas de aprendizagem em que os alunos estiveram empenhados e a qualidade de execução dessas tarefas, ao longo de uma unidade dedicada ao ensino do voleibol. Perante os resultados deste estudo, a autora evidencia a necessidade de as tarefas de aprendizagem serem estudadas de forma mais minuciosa pela investigação, na medida em que interferem quantitativa e qualitativamente nos efeitos da aprendizagem dos alunos de diferentes níveis de desempenho. Blakemore et al. (4) e Harrison et al. (24) desenvolveram estudos experimentais com o propósito de testar a organização do ensino de acordo com os princípios do mastery learning (dividir a unidade didáctica em sub-unidades e definir objectivos operacionais claros; avaliação formativa e informação sobre os progressos a par e passo; instrução adicional, reensino para os alunos que não atingiram os critérios da subunidade e passagem para nova sub-unidade apenas quando $80 \%$ do grupo cumpre os critérios de mestria; ênfase na coesão de grupo e na realização dos objectivos de mestria predefinidos). A concepção subjacente a esta abordagem é a de que através da melhoria das habilidades se melhorará a capacidade de jogo dos alunos. O primeiro estudo (4), aplicado 
em basquetebol, incluiu 3 turmas de rapazes do $7^{\circ}$ ano de escolaridade. Uma turma aplicou um programa de mastery learning durante 6 semanas com 5 aulas de 50 minutos por semana, outra turma aplicou um programa, com a mesma duração, centrado no desenvolvimento das habilidades do jogo e a outra serviu de grupo de controlo, tendo apenas realizado o préteste e o pós-teste. $\mathrm{Na}$ avaliação das habilidades isoladas o grupo do programa mastery learning foi o único que obteve um progresso consistente do pré-teste para o pós-teste. Porém, os grupos não se distinguem na realização das habilidades em situação de jogo, o que pode pôr em causa a pertinência dos objectivos do próprio programa.

O segundo estudo (24), aplicado em voleibol, incluiu 6 turmas de estudantes universitários em experiência na modalidade. Três turmas aplicaram o programa mastery learning e as outras três, o programa de desenvolvimento sequencial de habilidades inspirado no modelo de Rink (54). Os programas foram aplicados 2 vezes por semana, durante 16 semanas. Os resultados evidenciaram efeitos positivos em ambos os programas, os estudantes melhoraram signicativamente os resultados nos testes de habilidades, nas medidas de auto-eficácia e em vários indicadores de participação no jogo. Nenhum dos programas, porém, se distinguiu significativamente nas medidas consideradas.

Num outro estudo anterior (23), também aplicado no voleibol (duas turmas de estudantes universitários principiantes na modalidade e 19 sessões), os autores compararam dois estilos de ensino do espectro de Mosston, comando (andamento da actividade directamente regulado pelo professor) e tarefa (fichas de tarefas prescritas pelo professor, andamento regulado pelos alunos) e reportaram diferenças na evolução das curvas da taxa de sucesso referentes à execução das habilidades em situações de exercitação. Os alunos de nível baixo progridem mais no passe com o estilo comando, mas, por outro lado, progridem mais no remate com o estilo tarefa. Ao nível do desempenho das habilidades na situação de jogo não há diferenças entre os estilos, assim como não as há ao nível da auto-eficácia.

Uma outra equipa de investigadores (14) investigou o efeito das progressões de exercitação em condições de controlo experimental mais rigorosas, mas também mais artificiais. $\mathrm{O}$ estudo debruçou-se sobre a aprendizagem de duas habilidades isoladas do Voleibol, o serviço e o passe de frente. 36 alunos de uma turma do $9^{\circ}$ ano de escolaridade foram distri- buídos aleatoriamente por três programas experimentais, mantendo-se constantes, o número de sessões $(n=6)$, o número de execuções de cada habilidade por sessão $(n=10)$ e, consequentemente, o número total de execuções por habilidade $(n=60)$. O primeiro grupo (grupo de progressão) praticou o serviço e o passe segundo quatro etapas de complexidade crescente. O segundo grupo (grupo critério) aplicava as mesmas etapas, mas os alunos só avançavam para a última etapa após obter $80 \%$ de sucesso nas duas primeiras. $O$ terceiro grupo (teste final) praticou exclusivamente o teste de AAPHERD (1967) para as habilidades de Voleibol. 17 alunos de outra turma constituíram o grupo de controlo, não praticaram as habilidades, tendo apenas realizado o pré-teste e o pós-teste.

Os resultados evidenciaram a ineficácia do teste final, enquanto meio de exercitação das habilidades. Apenas os grupos que aplicaram as etapas de progressões evoluíram significativamente do pré-teste para o pós-teste, o que justifica a aplicação de progressões como forma de proporcionar a aprendizagem. No entanto, os alunos de nível baixo de habilidade, independentemente do tipo de progressões, realizam poucos ou nenhuns progressos. Os progressos verificados nas etapas iniciais não permitiram garantir sucesso nas etapas ulteriores, o que vem comprovar a interacção dos níveis de desempenho com os efeitos das condições de prática. Os autores concluem que as sequências práticas com etapas de dificuldade crescente parecem ser eficazes apenas quando as tarefas de aprendizagem estão apropriadas aos níveis de desempenho dos alunos.

A mesma equipa de investigadores (56) realizou um segundo estudo para averiguar o efeito conjugado das progressões com a utilização de refinamento das tarefas. Para o efeito, utilizou um protocolo com características semelhantes à do estudo anterior. Mesmo tipo de alunos, mesmos conteúdos de aprendizagem, mesmas etapas de progressões, mesmo número de ensaios, 10 ensaios por dia, durante 6 dias para a aprendizagem do passe e do serviço, tendo sido constituídos 5 grupos de alunos: (a) um grupo de controlo; (b) um grupo de progressão, sem refinamento; (c) um grupo de progressão com refinamento; (d) um grupo de teste final, sem refinamento (feedback motivacional); (e) um grupo de teste final com refinamento. As tarefas de refinamento surgiam após a realização de 5 ensaios e incidiam sobre os aspectos críticos de execução a ter em conta nos ensaios seguintes. Com base nos resulta- 
dos do pré-teste constituíram-se subgrupos por níveis de desempenho (fraco e moderado).

Os resultados corroboram, de uma forma geral, os encontrados no estudo anteriormente apresentado, uma vantagem clara dos grupos que trabalharam com progressões sobre os grupos que praticaram o teste final. Estes resultados evidenciaram ainda que, entre os dois grupos que foram sujeitos a etapas de progressão, o que beneficiou de refinamento das tarefas progrediu mais e obteve taxas de sucesso mais elevadas durante o período de instrução, embora esta constatação não tenha sido extensiva a todas as tarefas realizadas. Os autores reafirmam a necessidade de serem utilizadas progressões nas aprendizagens e a vantagem de refinamento das tarefas, em especial quando os alunos não conseguem fazer os ajustamentos necessários às exigências das novas situações de prática. Os autores verificaram novamente que vários alunos de nível de baixo de habilidade, independentemente do grupo em que trabalharam, não evoluíram do pré-teste para o pós-teste, indiciando que o objectivo de instrução, consubstanciado nas exigências do teste final, possa ser demasiado complexo e muito pouco consentâneo com as possibilidades facultadas pelos níveis de desempenho destes alunos.

Mesquita (39) num estudo aplicado no treino em Voleibol, no escalão de iniciados feminino, pretendeu analisar o efeito diferenciador da prática de tarefas estruturadas de forma progressiva em referência às exigências colocadas no jogo $2 \times 2$ e da informação orientada para os critérios de execução, sobre os ganhos na aprendizagem das habilidades técnicas. A amostra foi constituída por 21 jogadoras pertencentes a 3 equipas do referido escalão. $O$ estudo foi aplicado durante 18 sessões de treino, perfazendo na totalidade 56 sessões de treino com a duração de dois meses.

As 3 equipas foram sujeitas a programas de treino distintos: uma equipa aplicou o programa experimental de treino assente em progressões para a aprendizagem das habilidades de acordo com as exigências do jogo $2 \times 2$, fazendo-se acompanhar de informação focalizada nos critérios de realização das habilidades (eficiência) a ser ministrada pelo treinador; uma outra equipa aplicou o mesmo programa experimental de treino mas sem orientação ao nível da informação a ser ministrada pelo treinador; e uma terceira equipa serviu de grupo de controlo. Os resultados mostraram que o grupo de controlo que aplicou o jogo $6 \times 6$ como forma fundamental de apli- cação das habilidades em associação à exercitação de tarefas analíticas apenas registou alguns progressos no serviço. Dos grupos experimentais o que registou maiores ganhos na aprendizagem foi o que praticou tarefas estruturadas de forma progressiva em referência às exigências do jogo $2 \times 2$ e que usufruiu complementarmente de informação orientada para a qualidade de execução das habilidades (eficiência). Todavia, em termos absolutos, a variável que mais influência exerceu sobre os ganhos na aprendizagem foi o treino das habilidades em situações de prática sujeitas a uma interferência contextual moderada, patentes nas tarefas com estrutura funcional idêntica à do jogo $2 \times 2$. Perante estes resultados a autora realça a necessidade do treino das habilidades técnicas ser realizado em situações, que sem deixarem de integrar a lógica acontecimental do jogo e a sua estrutura funcional, devem facilitar o processo de aprendizagem das habilidades técnicas, principalmente nas de carácter aberto, apanágio dos jogos desportivos.

\section{CONDIÇÕES DE PRÁTICA E INTERFERÊNCIA CONTEXTUAL}

Vários estudos realizados em condições laboratoriais, no âmbito da Aprendizagem Motora, conceptualmente alicerçados na teoria da interferência contextual (Battig, 1966 apud (72), têm vindo a demonstrar a forte transferência produzida pela prática sujeita a elevada interferência contextual (32, 58), reportando que a prática das habilidades em condições variáveis é mais eficaz do que em condições constantes (66). A prática variável (com elevada interferência contextual) consiste em praticar uma habilidade em condições diferentes após cada ensaio ou após uma série de ensaios, em oposição à prática constante (com baixa interferência contextual) na qual as condições de realização são idênticas em todos os ensaios.

Admite-se que a variação das condições de prática pode levar a um maior esforço de processamento que se traduz numa menor performance durante a fase de aquisição, mas que pode ser recompensada nas fases de retenção e transferência. Todavia, Meira e Tani (37) não confirmaram o efeito da interferência contextual, quando se estendia a quantidade de prática, e admitem que ele não seja extensivo a todos os tipos de aprendizagem. Entretanto, como nos diz Rink (55): Se pode haver mérito em desenvolver um certo nível de consistência de performance em determinadas fases da aprendizagem, na maior parte da situações a 
repetição do mesmo movimento desencoraja níveis elevados de processamento, e no caso das habilidades abertas reduz a variabilidade de prática essencial para preparar o aluno para aplicar as habilidades num contexto mais complexo. (p. 6)

Ou seja, para que exista consistência no domínio de determinado comportamento motor é necessário que as condições de prática integrem variabilidade de situações congruentes com as exigências colocadas pelas situações de aplicação.

Se em condições laboratoriais os resultados tendem a reiterar, com algumas ressalvas, o efeito da interferência contextual, importa conferir autenticidade aos fenómenos através de estudos que, progressivamente, se aproximem dos envolvimentos reais de aprendizagem (34).

Goode e Magill (17) realizaram o primeiro estudo em condições não laboratoriais, embora sujeitas a elevado controlo, relativo à aprendizagem do serviço na modalidade de badminton. Constataram que o grupo sujeito a prática com elevada interferência contextual obteve melhores resultados nos testes de retenção e de transferência relativamente ao grupo que praticou sempre esta habilidade em condições de baixa interferência contextual.

Posteriormente, Wrisberg (72), através de um estudo realizado em condições de envolvimento típicas de um contexto de instrução real, pretendeu averiguar a integração de condições de prática variável em situações de ensino do serviço de badminton e a sua influência nos resultados da aprendizagem. Assim, constatou que o grupo sujeito a condições de prática com elevada interferência contextual evidenciou níveis de retenção significativamente superiores no serviço curto, em relação aos restantes grupos. Para além disso, os grupos que praticaram apenas uma versão de execução do serviço curto e longo e com elevada variabilidade contextual evidenciaram níveis de retenção significativamente superiores, relativamente aos grupos que praticaram três versões para cada tipo de serviço em condições de prática com baixa interferência contextual.

As diferenças de desempenho registadas no teste de retenção entre o serviço curto e longo $(17,72)$ evidenciam a influência distinta dos efeitos da prática variável na aquisição desta habilidade. As condições de execução da própria habilidade, ou seja, a distância de realização da habilidade ao alvo, parece interferir com o desempenho obtido, devendo tal situação ser contemplada na organização das tarefas de aprendizagem (72).
Num estudo mais longo, 30 ensaios ao longo de 9 aulas, aplicado ao ensino de 3 habilidades básicas do voleibol, serviço, passe e manchete, em condições reais de ensino, envolvendo 3 turmas subdivididas aleatoriamente em 3 grupos de prática com interferência contextual distinta, French, Rink e Werner (15) verificaram o progresso de todos os grupos, mas nenhum efeito distinto da interferência contextual. Num estudo semelhante Bortoli et al. (5) conseguiram evidenciar o efeito da interferência contextual apenas numa das habilidades, o serviço.

Parece não ser muito claro o panorama da transposição do efeito da interferência contextual para situações de prática aplicada. Brady (6) não observa qualquer efeito num estudo aplicado ao golfe. Farrow e Maschette (13) estudaram uma habilidade do ténis e registaram o efeito da interferência contextual apenas no grupo 10-12 anos, não extensivo ao grupo 810 anos. Também no ténis, Hebert, Landin e Solmon (25) verificaram que os sujeitos de menor nível de habilidade beneficiaram mais com a prática de baixa interferência contextual, não havendo diferenças entre os sujeitos de nível elevado. Entretanto outros estudos que se debruçaram sobre a aprendizagem de habilidades de batimento observaram a presença do efeito da interferência contextual nos ganhos das aprendizagens $(21,22)$. Landin e Hebert (30) lançam novas pistas para a avaliação do efeito da interferência contextual ao consideraram uma modelação dos níveis de interferência. Num estudo aplicado no Basquetebol, os autores constataram que tarefas com níveis extremos de interferência contextual (elevado e baixo) produzem menor efeito sobre as aprendizagens após o período de instrução (teste de retenção) relativamente a tarefas com níveis moderados de interferência contextual.

Como conclusão essencial destes estudos emerge a ideia de que a interferência contextual poderá estar associada à necessidade de reconstrução de planos de acção (32). As diferenças no período de aquisição normalmente observadas entre os grupos de prática com interferência contextual distinta podem estar, em parte, associadas aos processos de planeamento extra, realizados pelos sujeitos dos grupos de elevada interferência contextual (28). Em suma, a utilização de situações variadas de prática, per se, não permite a obtenção de níveis de desempenho superiores, se essas situações não oferecerem algo de diferente e estimulante do ponto de vista da aprendizagem $(33,70,71,72)$. 


\section{PARA ALÉM DA EXECUÇÃO DAS HABILIDADES BÁSICAS}

A identificação dos factores de eficácia dos programas, a determinação das variáveis ou conjunto de variáveis mais fortemente associadas ao sucesso da aprendizagem das habilidades do jogo têm sido a razão primeira para o esforço de investigação neste domínio.

O tempo e a oportunidade de resposta dos alunos com um determinado conteúdo constituíram temas privilegiados da agenda da investigação no ensino da educação física, muito particularmente entre os finais dos anos 1970 a meados dos anos 1990. Alguns desses estudos tomaram as habilidades básicas dos jogos desportivos como conteúdos de referência. A evolução da investigação nestes temas processou-se no sentido de uma crescente procura de qualificação e diferenciação do tempo de prática e oportunidade de resposta dos alunos. "O que no princípio começou por ser um conceito de tempo destinado pelo professor para cobrir uma dada matéria, foi sendo progressivamente refinado passando a incluir as ideias de sucesso dos alunos, conteúdo apropriado e mais recentemente níveis elevados de empenhamento do aluno na tarefa" (55, p. 4). Como estruturar, combinar, encadear os conteúdos da actividade, como variar as condições de prática, como dosear as tarefas de aprendizagem (quantidade e complexidade), como regular a progressão das aprendizagens para os diferentes tipos de alunos (objectivos, critérios, níveis e etapas), como apoiar o aluno nas suas dificuldades de aprendizagem e como orientá-lo para um nível de confronto óptimo com as tarefas de aprendizagem são questões que vêm sendo sucessivamente colocadas, que têm obtido algumas respostas valiosas para a orientação de uma prática de ensino, revelando factores associados ao sucesso de aprendizagem das habilidades. Outras questões há que permanecem sem um desfecho claro e outras ainda que carecem de ferramentas teóricas e de abordagens mais robustas para produzirem resultados mais convincentes ou esclarecedores.

A preocupação com o domínio das habilidades básicas tem norteado a maior parte do labor didáctico relacionado com o ensino dos jogos desportivos. A investigação nesta área tem em grande parte acolhido esta perspectiva, porquanto, as variáveis dependentes, os principais objectivos dos programas, os conteúdos de aprendizagem e os instrumentos de avaliação predominantemente utilizados têm tido como horizonte as habilidades básicas dos jogos.
No entanto, nos jogos desportivos colectivos, a realização superior das acções de jogo reclama um domínio das habilidades técnicas que possa dar suporte à concretização de uma "dupla tarefa", que se traduz na capacidade do praticante deslocar a atenção de uma tarefa motora (e.g., drible) para uma tarefa cognitiva (e.g., analisar os deslocamentos dos colegas na situação) (66).

Vankersschaver (68) refere que a evolução nessa dupla tarefa, ao nível das etapas de formação, é caracterizada pela passagem progressiva do controlo visual ao controlo quinestésico. Através do refinamento técnico, o jogador efectua, progressivamente, a passagem da atenção centrada na execução motora para os aspectos de ordem decisional (31). Ao prevalecer, na relação com a bola, o controlo quinestésico, o jogador tecnicamente evoluído fica liberto para efectuar a "leitura" das situações de jogo e, consequentemente, poder optar pelas melhores soluções. A questão que se coloca, do ponto de vista da organização do processo de aprendizagem, reside em decidir se é preferível iniciar a aprendizagem pela automatização da tarefa motora isoladamente, ou se é preferível confrontar directamente o praticante com as situações de "dupla tarefa", as quais exigem a prática das habilidades em condições de resposta aberta (66).

A este respeito, Anderson (2) sublinha que as tarefas de aprendizagem podem ser vistas como lugares para aplicar procedimentos algorítmicos a problemas com uma única resposta correcta, ou podem ser vistas como situações que obriguem os alunos a definir e a representar problemas e a transformar o conhecimento existente numa das muitas soluções possíveis. Na medida em que nos JDC as habilidades abertas predominam, as tarefas de aprendizagem mais adequadas devem responder a este tipo de exigências. Devem, por isso, envolver processos adaptativos, de forma a possibilitar a interpretação imediata do inesperado e assim proporcionar a tomada de decisões atempadas e oportunas (67).

De acordo com Rink (54), devido ao facto das habilidades técnicas nos JDC serem fundamentalmente de natureza aberta, assumindo um carácter multidimensional, a estruturação das tarefas deve contemplar os seguintes pressupostos:

(a) as habilidades devem ser praticadas em contextos variáveis com a solicitação de execuções variadas, o mais cedo possível; 
(b) as habilidades devem ser praticadas em contextos com baixa interferência contextual o menor tempo possível;

(c) devem ser privilegiadas as situações que coloquem problemas semelhantes aos que surgem em situação de jogo.

\section{CORRESPONDÊNCIA}

\section{Amândio Graça}

Faculdade de Ciências do Desporto

e de Educação Física

Rua Dr. Plácido Costa, 91

4200.450 Porto, Portugal

agraca@fcdef.up.pt 


\section{REFERÊNCIAS BIBLIOGRÁFICAS}

1. Alexander, K. (1983). Beyond the prediction of student achievement: Direct and repeated measurement of behavior change. Journal of Teaching in Physical Education Monog 1 Summer: 42-47.

2. Anderson, L. (1989). Implementing instructional programs to promote meaningful, self-regulated learning. In Brophy, J. (ed.). Advances in research on teaching. Greenwich, Conneticut: JAI Press inc. 311-343.

3. Anderson, W.G., Barrette, G.T. (1978). What's going on in gym: Descriptive studies of physical education classes. Newtown, Ct.: Motor skills.

4. Blakemore, C.L., Hilton, H.G., Harrison, J.M., Pellett, T.L., Gresh, J. (1992). Comparison of students taught basketball skills using mastery and nonmastery learning-methods. Journal of Teaching in Physical Education 11: 235-247.

5. Bortoli, L., Robazza, C., Durigon, V., Carra, C. (1992). Effects of contextual interference on learning technical sports skills. Perceptual and Motor Skills 75: 555-562.

6. Brady, F. (1997). Contextual interference and teaching golf skills. Perceptual and Motor Skills 84: 347-350.

7. Brooker, R., Kirk, D., Braiuka, S., Bransgrove, A. (2000) Implementing a game sense approach to teaching junior high school basketball in a naturalistic setting. European Physical Education Review 6: 7-25.

8. Brunelle, J., Godbout, P., Tousignant, M., Brunelle, J.P., Trudel, P. (1985). Relations between initial ability level, academic learning time and level of achievement of the program participants. Revue Quebecoise de L'activite Physique 3: 92-100.

9. Buck, M., Harrison, J.M. (1990). An analysis of game play in volleyball. Journal of Teaching in Physical Education(Champaign, Ill.) 10(11), Oct 1990, 38-48 Refs:7.

10. Buck, M., Harrison, J.M., Bryce, G.R. (1991). An analysis of learning trials and their relationship to achievement in volleyball. Journal of Teaching in Physical Education(Champaign, Ill.) 10(2), Jan 1991, 134-152 Refs:17.

11. Capel, S., Katene, W. (2000). Secondary pgce pe students' perceptions of their subject knowledge. European Physical Education Review 6: 46-70.

12. Carreiro da Costa, F. (1995). O sucesso pedagógico em educação física: Estudo das condições e factores de ensino-aprendizagem associados ao êxito numa unidade de ensino. Lisboa: Edições FMH.

13. Farrow, D., Maschette, W. (1997). The effects of contextual interference on children learning forehand tennis groundstrokes. Journal of Human Movement Studies 33: 47-67.

14. French, K.E., Rink, J.E., Rikard, L., Mays, A., Lynn, S., Werner, P. (1991). The effects of practice progressions on learning two volleyball skills. Journal of Teaching in Physical Education-(Champaign, Ill.) 10(3), Apr 1991, 261-274 Refs: 26 .

15. French, K.E., Rink, J.E., Werner, P.H. (1990). Effects of contextual interference on retention of 3 volleyball skills. Perceptual and Motor Skills 71: 179-186.

16. Godbout, P., Brunelle, J., Tousignant, M. (1987). Who benefits from passing through the program? In Barrette, G.T. (ed.). Myths, models, and methods in sport pedagogy. Champaign, Ill: Human Kinetics Publishers. 183-197.

17. Goode, S., Magill, R.A. (1986). Contextual interference effects in learning three badminton serves. Research Quarterly for Exercise and Sport-(Reston, Va.) 57(4), Dec 1986, 308-314.
18. Graça, A. (1991). O tempo e a oportunidade para aprender o basquetebol na escola: Análise de uma unidade de ensino com alunos do $5^{\circ}$ ano de escolaridade. Universidade do Porto: Faculdade de Ciências do Desporto e de Educação Física.

19. Graça, A. (1997). O conhecimento pedagógico do conteúdo no ensino do basquetebol. Dissertação de Doutoramento: Universidade do Porto.

20. Graham, K. (1987). A description of academic work and student performance during a middle school volleyball unit. Journal of Teaching in Physical Education 7: 22-37.

21. Green, D.P., Whitehead, J., Sugden, D.A. (1995). Practice variability and transfer of a racket skill. Perceptual and Motor Skills 81: 1275-1281.

22. Hall, K.G., Domingues, D.A., Cavazos, R. (1994). Contextual interference effects with skilled baseball players. Perceptual and Motor Skills 78: 835-841.

23. Harrison, J., Fellingham, G., Buck, M., Pellet, T. (1995). Effects of practice and command styles on rate of change in volleyball performance and self efficacy of high-, medium, and low-skilled learners. Journal of Teaching in Physical Education 14: 328-339.

24. Harrison, J.M., Preece, L.A., Blakemore, C.L., Richards, R.P., Wilkinson, C., Fellingham, G.W. (1999). Effects of two instructional models-skill teaching and mastery learning-non skill development, knowledge, self-efficacy, game play, volleyball. Journal of Teaching in Physical Education 19: 34-57.

25. Hebert, E.P., Landin, D., Solmon, M.A. (1996). Practice schedule effects on the performance and learning of lowand high-skilled students: An applied study. Research Quarterly for Exercise and Sport 67: 52-58.

26. Holt, N.L., Strean, W.B., Bengoechea, E.G. (2002). Expanding the teaching games for understanding model: New avenues for future research and practice. Journal of Teaching in Physical Education 21: 162-176.

27. Housner, L. (1990). Selecting master teachers: Evidence from process-product research. Journal of Teaching in Physical Education 9: 201-226.

28. Immink, M.A., Wright, D.L. (1998). Contextual interference: A response planning account. Quarterly Journal of Experimental Psychology Section a-Human Experimental Psychology 51: 735-754.

29. Laakso, L., Telama, R., Varstala, V. (1985). Team game observations in finnish physical education classes. In P.F, R. (ed.).

30. Landin, D., Hebert, E. (1997). A comparison of three practice schedules along the contextual interference continuum. Resesarch Quarterly for Exercise and Sport, 68: 357-361.

31. Lassierra, G. (1990). Aproximacion a una propuesta de aprendizaje de los elementos tacticos individuales en los deportes de equipo. Apunts d' Educació Fisica i Esportes 24: 59-68.

32. Lee, T.D., Magill, R.A. (1983). Activity during the post-kr interval: Effects upon performance or learning? Research Quarterly for Exercise and Sport 54: 340-345.

33. Lee, T.D., Wishart, L.R., Cunningham, S., Carnahan, H. (1997). Modeled timing information during random practice eliminates the contextual interference effect. Research Quarterly for Exercise and Sport-(Reston, Va.) 68(1), Mar 1997, 100-105 Refs:12, Total Pages: 6.

34. Lidor, R. (1995). The implementation of constant and variable - practices in skill acquisition: A field study. International Journal of Physical Education (Schorndorf) 32: 11-16. 
35. Locke, L. (1981). Teaching and learning processes in physical activity: The central problem of sport pedagogy. In Haag, H. (ed.). XXII ICHPER World Congress. Kiel: Schorndorf, Verlag Karl Hoffmann. 140-154.

36. Mancini, V.H., Wuest, D.A. (1987). Coaches' interactions and their high- and low-skilled athletes' alt-pe: A systematic perspective. In Barrette, G.T. (ed.). Myths, models, and methods in sport pedagogy: Proceedings of the adelphi-aiesep '85 world sport conference (1985: Adelphi university).

Champaign, Ill: Human Kinetics Publishers. 231-237.

37. Meira, C.M., Tani, G. (2001). The contextual interference effect in acquisition of dart-throwing skill tested on a transfer test with extended trials. Perceptual and Motor Skills 92: 910-918.

38. Mesquita, I. (1996). Estudo descritivo e comparativo das respostas motoras de jovens voleibolistas de diferentes níveis de desempenho nas situações de treino e competição. Estudos CEJD 1: 32-56.

39. Mesquita, I. (1998). A instrução e a estruturação das tarefas no treino de voleibol: Estudo experimental no escalão de iniciados feminino. Dissertação de Doutoramento: Universidade do Porto.

40. Metzler, M. (1982). Adapting academic learning time instructional model tophysical education teaching. Journal of Teaching in Physical Education 1: 44-55.

41. Metzler, M. (1989). A review of research on time in sport pedagogy. Journal of Teaching in Physical Education(Champaign, Ill.) 8(2), Jan 1989, 87-103 Refs:81.

42. Nixon, J., Locke, L. (1973). Research on teaching in physical education. In Travers, M. (ed.). Handbook of research on teaching. Chicago: Rand McNally. 1210-1242.

43. Oslin, J., Mitchell, S., Griffin, L. (1998). The game performance assessment instrument (gpai): Development and preliminary validation. Journal of Teaching in Physical Education 17: 231-243.

44. O'Sullivan, M.M. (1985). A descriptive analytical study of student teacher effectiveness and student behaviour in secondary school physical education. In Howe, B.L., Jackson, J.J. (eds.). Teaching effectiveness research. Victoria, B.C: University of Victoria, School of Physical Education. 22-30.

45. Parker, M., O'Sullivan, M. (1983). Modifying alt-pe for game play contexts and other reflections. In Journal of Teaching in Physical Education, m.S.R. (ed.).

46. Phillips, D.A., Carlisle, C. (1983). Comparison of physical education teachers categorized as most and least effective. Journal of Teaching in Physical Education-(Champaign, Ill.) 10(1), Oct 1990, 76-83 Refs:23 2: 55-67.

47. Pieron, M. (1982). Behaviors of low and high achievers in physical education classes. In Pieron, M., Cheffers, J. (eds.). Studying the teaching in physical education. Liege: AISEP. 53-60.

48. Pieron, M., Dohogne, A. (1980). Comportements des eleves dans des classes d' education physique conduites par des enseignants en formation. Revue de l' Education Physique 20: 11-18.

49. Pieron, M., Forceille, C. (1983). Observation du comportement des élèves dans des classes de l'enseignement secondaire: Influence de leur niveau d'habilité. Revue de l'Education Physique 23: 9-16.

50. Pieron, M., Gonçalves, C. (1987). Participant engagement and teacher's feedback in physical education teaching and coaching. In Barrette, G.T. (ed.). Myths, models, and methods in sport pedagogy: Proceedings of the adelphi-aiesep ' 85 world sport conference (1985 : Adelphi university). Champaign, Ill: Human Kinetics Publishers. 249-254.

51. Piéron, M., Haan, J. (1981). Interactions between teachers and students in physical education setting: Observations of student behaviors. In Haag, H. (ed.). Proceedings of the xxii. Ichper-world-congress: Kiel 1979, july 23rd-27th, 1979. Kiel: Schorndorf, Verlag Karl Hoffmann. 364-368.

52. Pimentel, J. (1987). Jogos desportivos colectivos: Comportamento dos alunos mais dotados e menos dotados. Horizonte 21: 95-102.

53. Putnam, R., Lampert, M., Peterson, P. (1990). Alternative perspectives on knowing mathematics in elementary schools. In Cazden, C. (ed.). Review of research in education. Washington, DC: American Educational Research Association. 57-150.

54. Rink, J. (1993). Teaching physical education for learning. St. Louis: Mosby.

55. Rink, J. (1999). What do students learn in physical education and how do they learn? AIESEP Newsletter 63: 2-10.

56. Rink, J.E., French, K.E., Werner, P.H., Lynn, S., Mays, A. (1992). The influence of content development on the effectiveness of instruction. Journal of Teaching in Physical Education-(Champaign, Ill.) 11(2), Jan 1992, 139-149 Refs:24.

57. Rink, J.E., Werner, P. (1987). Student responses as a measure of teacher effectiveness. In Barrette, G.T. (ed.). Myths, models, and methods in sport pedagogy: Proceedings of the adelphiaiesep '85 world sport conference (1985 : Adelphi university). Champaign, Ill: Human Kinetics Publishers. 199-206.

58. Shea, J.B., Morgan, R.L. (1979). Contextual interference effects on the acquisition, retention, and transfer of a motor skill. Journal of Experimental Psychology: Human Learning and Memory 5: 179-187.

59. Shute, S., Dodds, P., Placek, J., Rife, F., Silverman, S. (1982). Academic learning time in elementary school movement education: A descriptive analytic study. Journal of Teaching in Physical Education 1: 3-14.

60. Siedentop, D. (1983). Academic learning time: Reflections and prospects. Journal of Teaching in Physical Education: 3-7.

61. Siedentop, D., Tannehill, D. (2000). Developing teaching skills in physical education. Mountain View, Calif.: Mayfield Pub. Co.

62. Silverman, S., Devillier, R., Ramirez, T. (1991). The validity of academic learning time-physical education (alt-pe) as a process measure of achievement. Research Quarterly for Exercise and Sport-(Reston, Va.) 62(4), Sept 1991, 319-325 Refs:34.

63. Telama, R., Paukku, P., Varstala, V., Paananen, M. (1982). Pupils' physical activity and learning behaviour in physical education classes. In Pieron, M., Cheffers, J. (eds.). Studying the teaching in physical education. Liege: AISEP. 22-35.

64. Telama, R., Varstala, V., Heikinaro-, Johansson, P., Paukku, P. (1986). The relationship between pupil's leisure-time physical activity and motor behavior during physical education lessons. In Pieron, M., Graham, G. (eds.). Sport pedagogy: Proceedings of the olympic scientific congress (1984: Eugene, ore. Champaign, Ill: Human Kinetics Publishers. 57-62.

65. Telama, R., Varstala, V., Paukku, P., Paananen, M., Heikinaro-Johansson, P. (1985). Pupils' learning behaviors and activity models in team sport lessons from pedagogical point of view. In P.F, R. (ed.). 
66. Temprado, J.-J. (1997). Apprentissage moteur: Quelques données actuelles. Education Physique et Sport 267: 20-23.

67. Turner, A., Martinek, T. (1995). Teaching for understanding: A model for improving decision making during game play. Quest 47: 44-63.

68. Vankersschaver, J. (1983). Analyse d'un geste technique au football en situation de double tache visuelle. Motricite Humaine 1: 29-38.

69. Varstala, V., Telama, R., Paukku, P., Heikinaro-Johansson, P. (1985). An observational study of teaching team games in school physical education lessons. In P.F, R. (ed.).

70. Wright, D.L., Coady, W. (1997). Cognitive processes related to contextual interference and observational learning: A replication of blandin, proteau, and alain (1994). Research Quarterly for Exercise and Sport-(Reston, Va.) 68(1), Mar 1997, 106-109 Refs:8, Total Pages: 4.

71. Wright, D.L., Smith-Munyon, L., Sidaway, B. (1997). How close is too close for precise knowledge of results? Research Quarterly for Exercise and Sport-(Reston, Va.) 68(2), June 1997, 172-176 Refs:17, Total Pages: 5.

72. Wrisberg, C.A. (1991). A field-test of the effect of contextual variety during skill acquisition. Journal of Teaching in Physical Education 11: 21-30. 